\title{
Superhero Rictor promotes cellular differentiation of mouse embryonic stem cells
}

\author{
Youming Zhu ${ }^{1} \cdot$ Peng Wang ${ }^{2} \cdot$ Li Zhang $\mathbb{1}^{3} \cdot$ Guo Bai $^{4} \cdot$ Chi Yang ${ }^{4} \cdot$ Yuanying Wang ${ }^{1} \cdot$ Jiacai He $^{1} \cdot$ Zhiyuan Zhang $^{4}$. \\ Guoping $\mathrm{Zhu}^{2} \cdot$ Duohong Zou ${ }^{1,4}$
}

Received: 20 November 2017 / Revised: 9 July 2018 / Accepted: 13 July 2018 / Published online: 28 August 2018

๑) ADMC Associazione Differenziamento e Morte Cellulare 2018

\begin{abstract}
Embryonic stem cells (ESCs) hold great promise for regenerative medicine. To harness the full therapeutic potential of ESCs, better understanding of the molecular mechanisms underlying the maintenance and differentiation of ESCs is required. Mammalian target of rapamycin (mTOR) is a serine/threonine protein kinase that integrates growth factor receptor signaling with cellular growth and proliferation. Dysregulation of mTOR signaling has been linked to various human diseases including cancer and metabolic syndromes. However, little is known regarding the function of mTOR signaling in the regulation of ES cell differentiation. Here we report that Rictor, a key component of mTORC2, functions as a novel ES cell differentiation promoting factor. Mechanistically, Rictor is able to interact with Prkch and facilitate Prkch phosphorylation at Ser-642. Upon phosphorylation, Prkch promotes Klf4 phosphorylation and inhibits Klf4-dependent Ecadherin expression, thereafter leading to the ES cell differentiation. These findings reveal a novel Rictor-Prkch-Klf4 pathway that plays an important role in the regulation of ES cell differentiation.
\end{abstract}

Edited by E. Baehrecke

These authors contributed equally: Youming Zhu, Peng Wang

Electronic supplementary material The online version of this article (https://doi.org/10.1038/s41418-018-0177-5) contains supplementary material, which is available to authorized users.

Guoping Zhu

gpz1996@yahoo.com

$\triangle$ Duohong Zou

zdhyy@ahmu.edu.cn

1 Department of Dental Implant Center, Stomatologic Hospital \& College, Anhui Medical University, Key Laboratory of Oral Diseases Research of Anhui Province, Hefei, Anhui Province 230022, China

2 Institute of Molecular Biology and Biotechnology, College of Life Sciences, Anhui Normal University, No. 1 Beijing East Road, Wuhu, Anhui Province 241000, China

3 Department of Urology, The First Affiliated Hospital of Anhui Medical University, Anhui Medical University, Hefei, Anhui Province 230022, China

4 Department of Oral Surgery, Ninth People's Hospital, College of Stomatology, Shanghai Jiao Tong University School of Medicine; National Clinical Research Center for Oral Disease; Shanghai Key Laboratory of Stomatology \& Shanghai Research Institute of Stomatology, Shanghai 200001, China

\section{Introduction}

Embryonic stem cells (ESCs) are distinguished by their abilities to self-renew and to differentiate into various types of cells, and have great potential for regenerative medicine [1-3]. The fate of ESCs is delicately regulated by a complex network formed by transcriptional factors, epigenetic regulators, signal receptors, and protein kinases. In mouse ESCs, the maintenance of self-renewal is primarily regulated by LIF-STAT3 $[4,5]$ and BMP pathway [6], while the differentiation of ESCs is promoted by MEK-ERK and Gsk3 $\beta$ signaling [7]. The downstream transcription factors Oct4, Sox 2 and Nanog work as a central complex to activate genes that sustain the undifferentiated state and suppress genes that promote differentiation $[8,9]$.

The mammalian target of rapamycin (mTOR) signaling regulates cell proliferation, cell survival, and protein synthesis $[10,11]$. There are two distinct types of mTOR complexes, mTORC1 and mTORC2. mTORC1 is composed of mTOR, Raptor, MLST8, PRAS40, and DEPTOR [12]. The complex is activated by insulin, growth factors, and oxidative stress, and regulates protein translation through its substrates 4E-BP1 and S6K1. mTORC2 mainly consists of mTOR, rapamycin-insensitive companion of mTOR (Rictor), MLST8, and mSIN1 [13], and responds to 

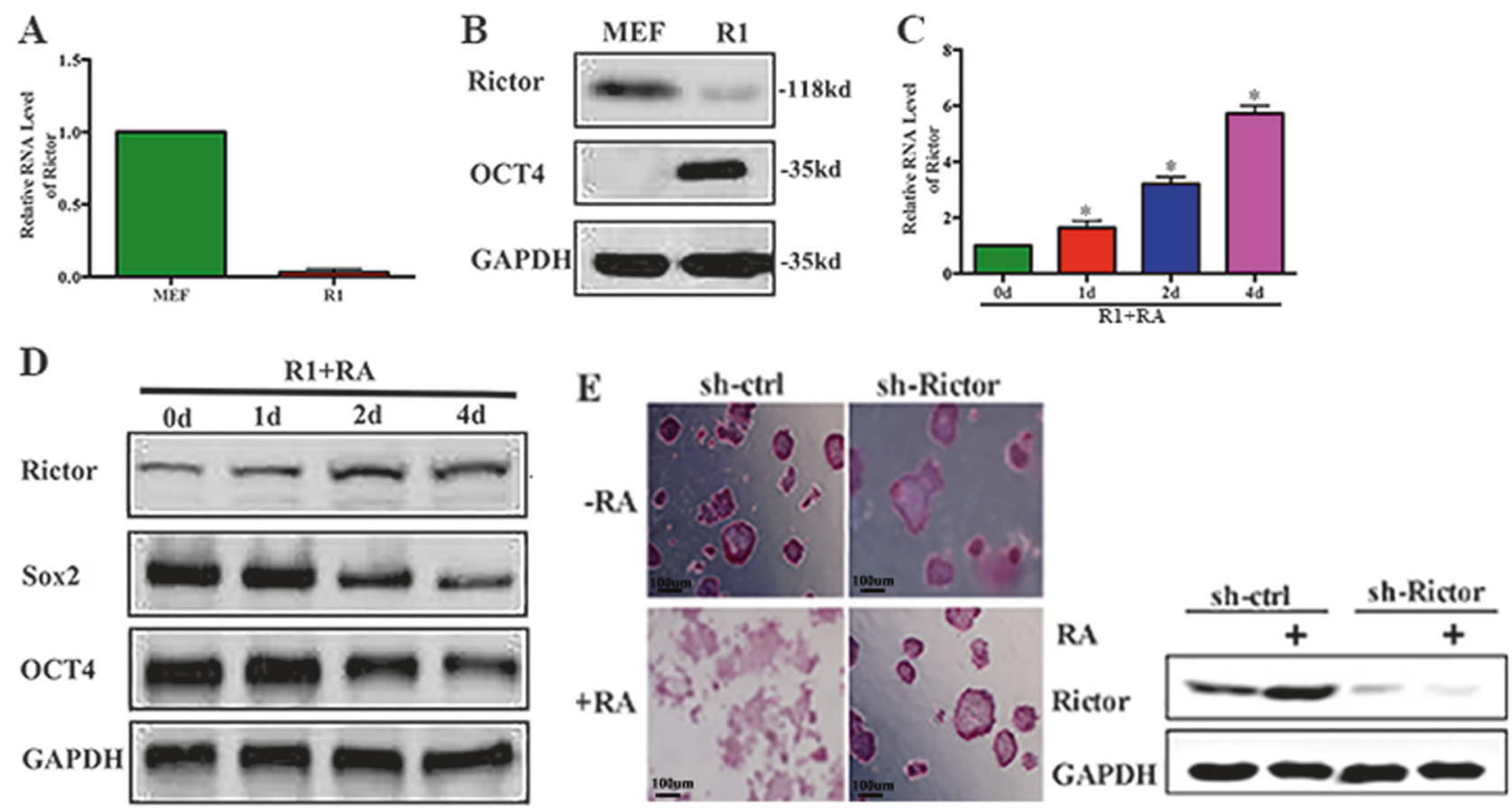

F
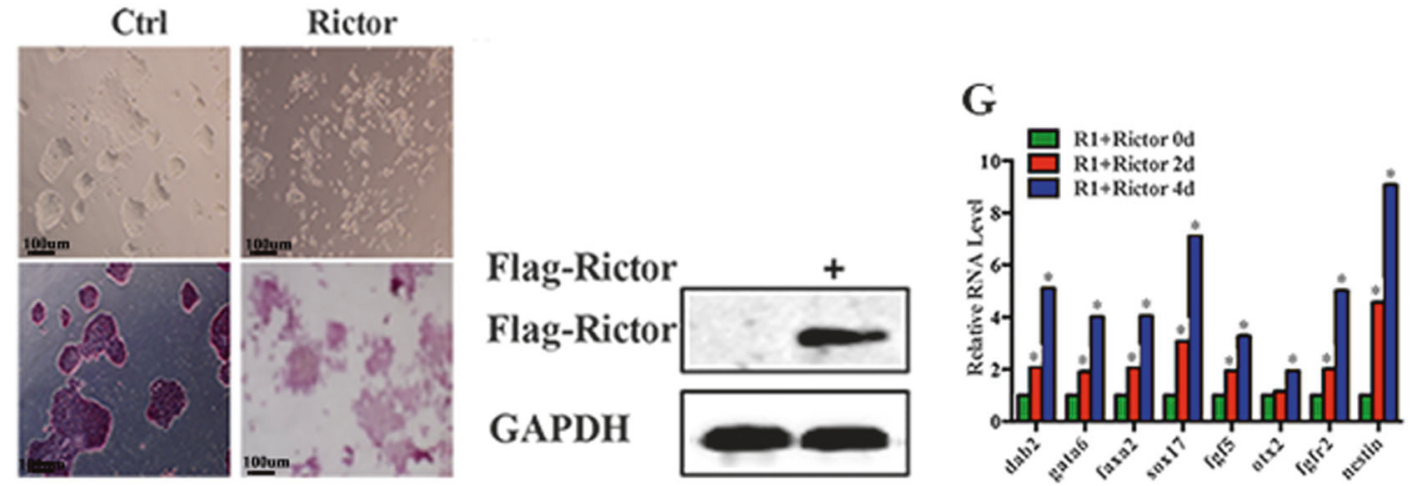

Fig. 1 Rictor promotes ES cell differentiation. a Real-time RT-PCR analysis of Rictor mRNA expression in MEF and R1 cells. Data are shown as mean \pm SD of three replications. b Lysates from MEF or $\mathrm{R} 1$ cells were analyzed by western blot with the indicated antibodies. $\mathbf{c}$ $\mathrm{R} 1$ cells were treated with $0.3 \mu \mathrm{M}$ RA for the indicated periods of time. Total RNA was extracted and subjected to real-time RT-PCR analysis to examine Rictor mRNA expression. d R1 cells were treated with 0.3 $\mu \mathrm{M}$ RA for the indicated periods of time. Cell lysates were analyzed by western blot with the indicated antibodies. e R1 mouse ES cells expressing control- or Rictor-specific shRNAs were treated with 0.3

$\mu \mathrm{M}$ RA for 4 days. The ES cell colonies were stained for alkaline phosphotase activity. The shown images are representative from three replications. f R1 cells were infected with lentiviruses expressing either control or Rictor proteins. Six days after infection, the ES cell colonies were stained for alkaline phosphotase activity. The shown images are representative from three replications. $g$ R1 cells were infected with lentiviruses expressing Rictor for the indicated periods of time. Total RNA was then extracted and subjected to real-time RTPCR analysis

Rapamycin only in certain cell types under prolonged exposure. The function of mTORC2 is well understood compared to mTORC1, yet increasing evidence suggests that mTORC2 plays an important role in cancer pathogenesis, promoting tumor growth and chemotherapy resistance in cancer cells [14, 15].

As a key component of mTORC2, Rictor was originally identified as an mTOR binding partner [16]. Rictor is also shown to interact with mSIN1 and Protor1, both of which exist in mTORC2 complex [17]. It has been reported that the Rictor-mTOR complex is able to phosphorylate Akt on Ser 473 and facilitate the phosphorylation of AKT on Thr 308 by PDK1 [18]. The Rictor-mTOR complex also regulates the phosphorylation of PKC $\alpha$, thereby modulating the actin cytoskeleton [16]. In breast cancer cells, Rictor binds to $\mathrm{PKC} \zeta$ and regulates cancer metastasis [19]. Loss of Rictor gene in mice leads to embryo growth arrest at embryonic day 11.5 (E11.5), indicating an important role of 
A
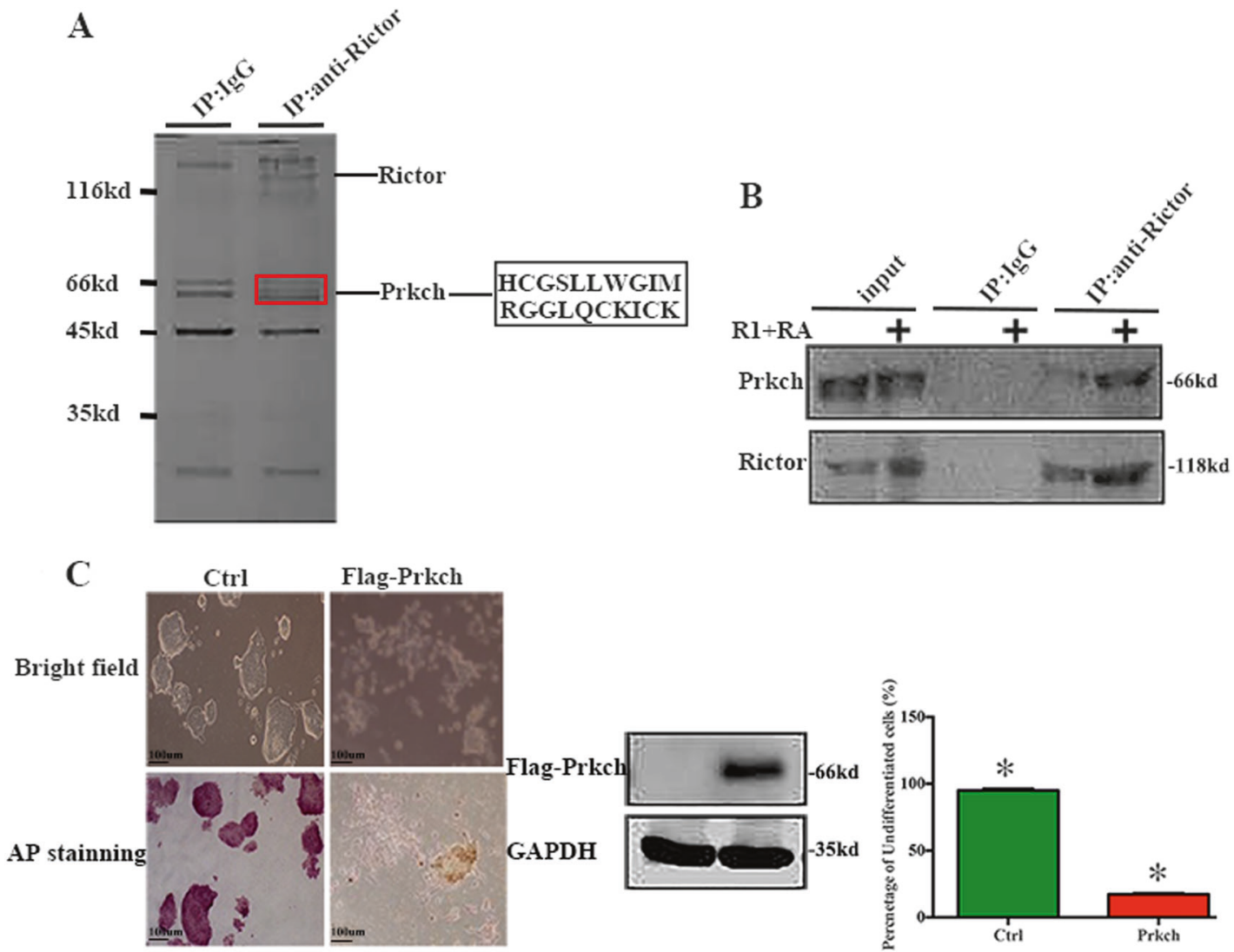

D
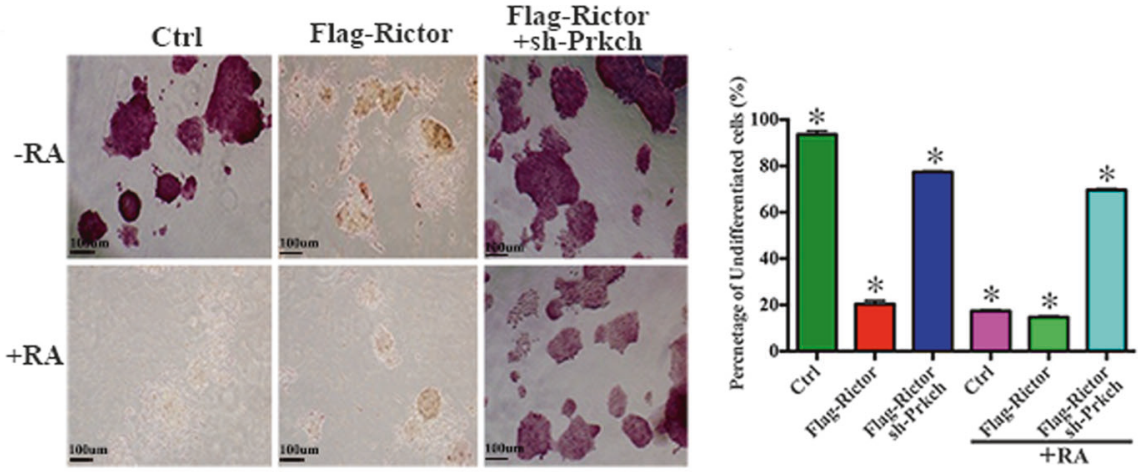

E
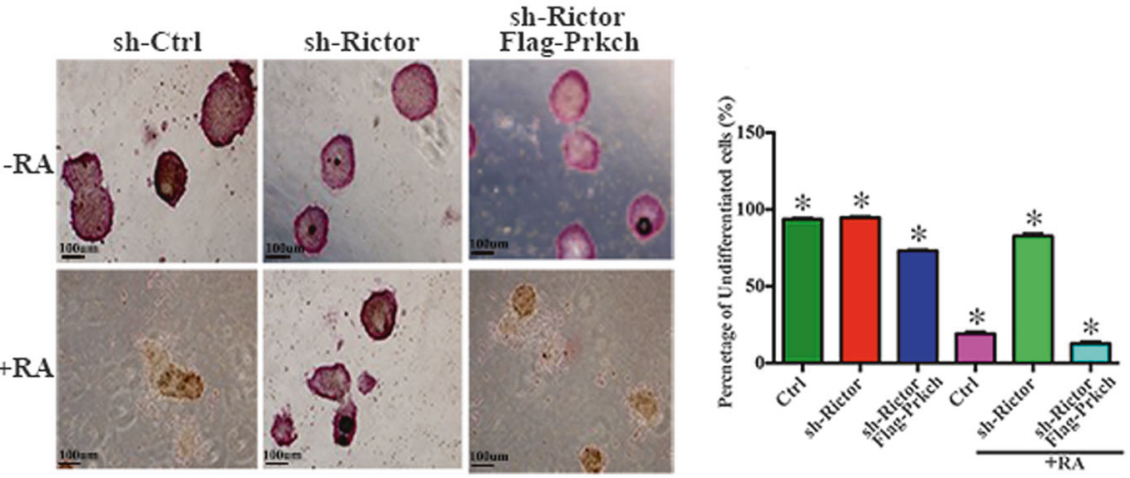

Rictor in the regulation of embryonic development [20]. However, the function of Rictor in embryonic stem cells has not been characterized.
Here we provide evidence that Rictor functions as a novel mouse ES cell differentiation promoting factor. Rictor exerts its function via interacting with Prkch and enhancing 
Fig. 2 Rictor promotes ES cell differentiation through Prkch. a R1 cells were treated with $0.3 \mu \mathrm{M}$ RA for 4 days. Cell lysates were then incubated with anti-Rictor antibody or an isotype-matched control IgG. The immunoprecipitates were resolved by SDS-PAGE and visualized by Coomassie brilliant blue staining. The indicated protein band was identified as Prkch by mass spectrometry. The obtained peptide sequences by mass spectrometry analysis were shown on the right. b R1 cells were treated with or without $0.3 \mu \mathrm{M}$ RA for 4 days. Cell lysates were immunoprecipitated with anti-Rictor antibody or an isotype-matched control IgG. The immunoprecipitates were analyzed by western blot with the indicated antibodies. $\mathbf{c}$ R1 cells were infected with lentiviruses expressing either Flag-Prkch or control. Six days after infection, the ES cell colonies were stained for alkaline phosphotase activity. The shown images are representative from three replications. d R1 cells expressing control or Flag-Rictor were infected with lentiviruses expressing Prkch-specific shRNAs or control as indicated. Two days after infection, cells were treated with or without $0.3 \mu \mathrm{M}$ RA for 4 days. The ES cell colonies were then stained for alkaline phosphotase activity. The shown images are representative from three replications. e R1 cells expressing control- or Rictor-specific shRNAs were infected with lentiviruses expressing Flag-Prkch or control as indicated. Two days after infection, cells were treated with or without $0.3 \mu \mathrm{M}$ RA for 4 days. The ES cell colonies were then stained for alkaline phosphotase activity. The shown images are representative from three replications

Prkch phosphorylation. Phosphorylated Prkch in turn increases phosphorylation of Klf4 and decreases its transcriptional activity toward E-cadherin. Therefore, our findings suggest that the Rictor-Prkch-Klf4 pathway has an important role in the regulation of ES cell differentiation.

\section{Results}

\section{Rictor promotes ES cell differentiation}

The mammalian target of rapamycin (mTOR) controls cell growth and proliferation in response to nutrients and growth factors through two distinct multiprotein complexes mTORC1 and mTORC2. As a key component of mTORC2, Rictor is essential for embryonic development, as evidenced by the finding that Rictor knockout leads to embryonic lethality in mice. However, it remains unknown if Rictor is involved in the regulation of maintenance or differentiation of mouse ES cells. To address this, we first compared expression of Rictor in MEF and R1 ES cells. Rictor was expressed at significantly lower levels in R1 cells compared to MEF cells (Fig. 1a, b). However, in response to retinoic acid (RA)-induced R1 ES cell differentiation, both mRNA and protein levels of Rictor were strongly upregulated (Fig. 1c, d). These data indicate that expression levels of Rictor are correlated with the differentiation state of ES cells.

We next evaluated the effect of Rictor on ES cell pluripotency maintenance. Knockdown of Rictor was shown to prevent R1 ES cells from RA-induced differentiation
(Fig. 1e). By contrast, exogenous expression of Rictor resulted in the differentiation of R1 ES cells, as manifested by both decreased colony genicity of R1 ES cells and increased expression of differentiation markers upon Rictor induction (Fig. 1f, g). We also confirmed that shRictor $\mathrm{R} 1$ cells transfected with the shRNA-insensitive version of Rictor can rescue RA-induced differentiation of R1 ES cells (Figure S1A). The induction of stem cell differentiation via Rictor has also been demonstrated in E14 ES cells (Figure S1B). Together, these results reveal an important function of Rictor in promoting mouse ES cell differentiation.

\section{Rictor promotes ES cell differentiation via Prkch}

To understand the underlying mechanism whereby Rictor promotes ES cell differentiation, we carried out an affinity purification approach to identify new Rictor-interacting partners. Lysates from R1 ES cells were incubated with anti-Rictor antibody or an isotype-matched control IgG, and the immunoprecipitated proteins were resolved by SDS-PAGE. The subsequent mass spectrometry analysis revealed Prkch as a Rictor-interacting candidate protein (Fig. 2a) and the main results of the LC-MS/MS analysis are shown in the supplementary table 1 . Prkch is a member of the PKC family proteins, and has been reported to regulate cell proliferation, apoptosis, and differentiation. To verify the interaction of Prkch with Rictor, we performed a co-immunoprecipitation experiment with anti-Rictor antibody. The results show that Prkch is able to interact with Rictor in R1 cells under both RA-treated and -untreated conditions. At the same time, we found that Rictor expression was upregulated and more Prkch was combined with Rictor under RA treatment (Fig. 2b). The increase in binding under RA treatment occurred not only with the upregulation of Rictor expression, but also concurrently with increased binding strength of Rictor and Prkch (Figure S2A).

We next evaluated the effect of Prkch on ES cell pluripotency maintenance. Exogenous expression of Prkch resulted in the differentiation of R1 ES cells, as manifested by decreased colony genicity of R1 ES cells (Fig. 2c). We also confirmed that transfection with shRNA Prkch can rescue the flag-Prkch, inducing $\mathrm{R} 1 \mathrm{ES}$ cells differentiation (Figure S2B), determining Rictor promotes ES cell differentiation through Prkch. The Rictor-initiated differentiation phenotype of R1 ES cell was rescued by knockdown of Prkch (Fig. 2d). In addition, Rictor knockdown consistently protected R1 ES cells from RA-induced differentiation; however, this was completely reversed by exogenous expression of Prkch (Fig. 2e). These data indicate that Prkch mediates the promoting effect of Rictor on ES cell differentiation. 
A

Flag-Rictor

P-Prkch

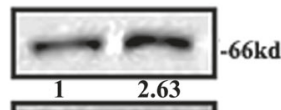

Prkch

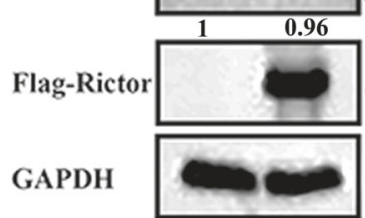

B

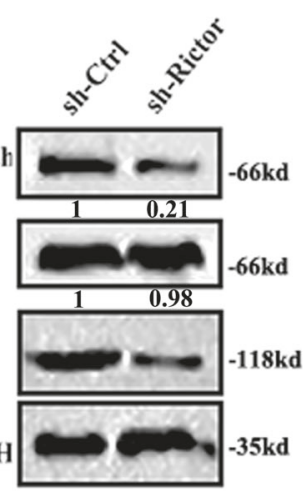

D

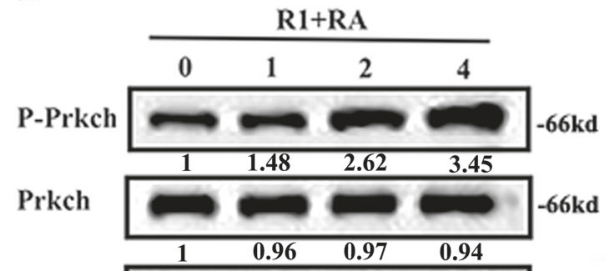

Rictor

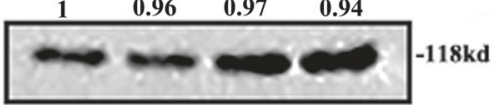

GAPDH

C
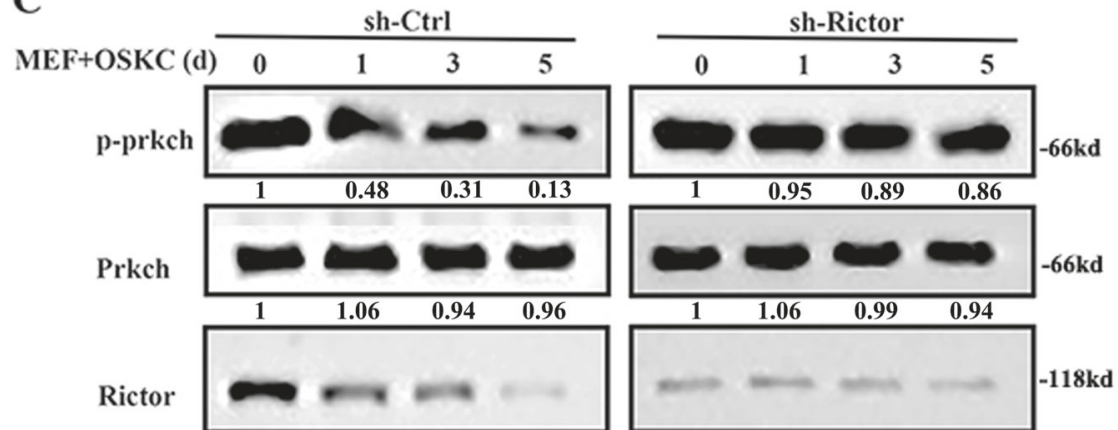

GAPDH
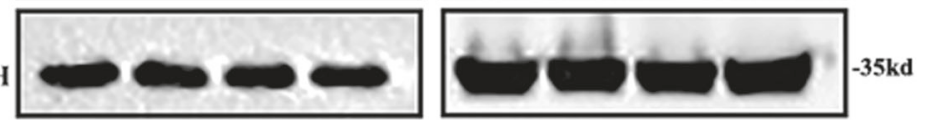

E
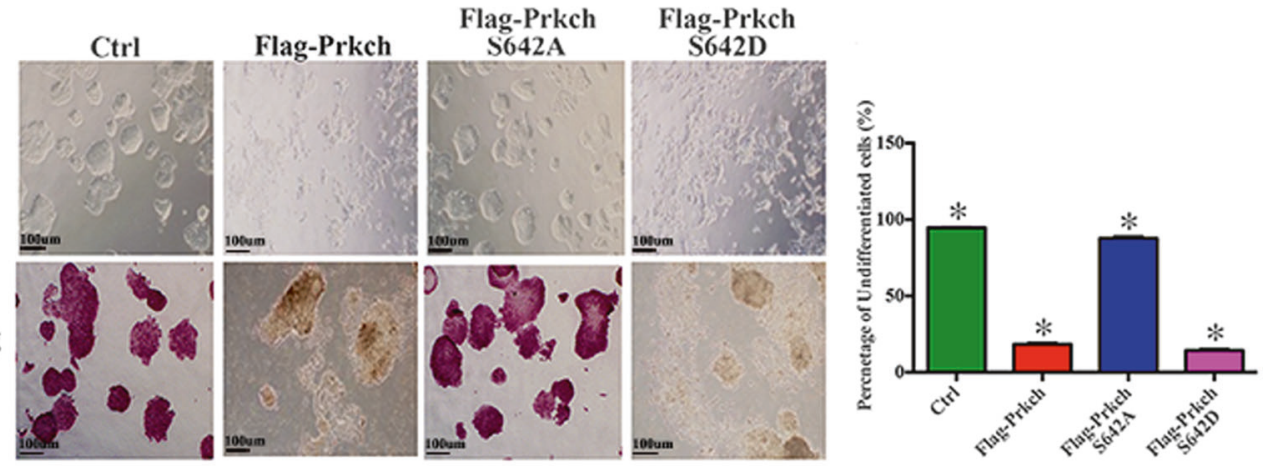

F

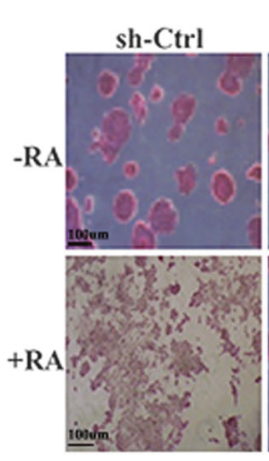

sh-Rictor

sh-Rictor
PrkchS642A

sh-Rictor
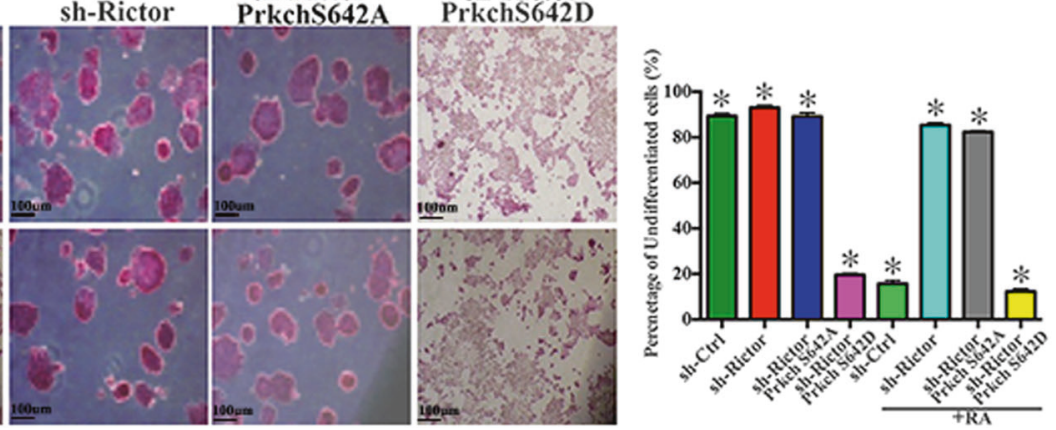
Fig. 3 Rictor promotes ES cell differentiation via increasing Prkch phosphorylation. a MEF cells were infected with lentiviruses expressing either control or Flag-Rictor protein. Fourty-eight hours after infection, cell lysates were analyzed by western blot with the indicated antibodies. b MEF cells were infected with lentiviruses expressing control- or Rictor-specific shRNAs. Fourty-eight hours after infection, cell lysates were analyzed by western blot. $\mathbf{c}$ Lysates from MEF cells on days $0,1,3$, and 5 after transduction with OSCK were analyzed by western blot with the indicated antibodies. $\mathbf{d}$ R1 cells were treated with $0.3 \mu \mathrm{M}$ RA for the indicated periods of time. Cell lysates were analyzed by western blot. e R1 cells were infected with lentiviruses expressing wild type Prkch or the indicated Prkch mutants. Six days after infection, the ES cell colonies were stained for alkaline phosphotase activity. The shown images are representative from three replications. $\mathbf{f} \mathrm{R} 1$ cells expressing the indicated shRNAs and proteins were treated with $0.3 \mu \mathrm{M}$ RA for 4 days. The ES cell colonies were then stained for alkaline phosphotase activity. The shown images are representative from three replications

\section{Rictor promotes ES cell differentiation through increasing Prkch phosphorylation}

It has been reported that mTORC2 modulates phosphorylation of Prkca at Ser-657. We therefore asked whether Rictor could regulate Prkch phosphorylation. Our data showed that ectopic expression of Rictor increased, whereas knockdown of Rictor decreased Prkch phosphorylation at Ser-642 in MEF cells (Fig. 3a, b). Torin1, a mTORC2 inhibitor, can downregulate the expression of p-Prkch (Figure S3A), and in vitro kinase assay also proved that MTORC2 directly phosphorylated Prkch (Figure S3B and C). In addition, along with the increasing expression of Rictor, levels of phosphorylated Prkch were concurrently increased in R1 ES cells upon RA-induced differentiation (Fig. 3c). We also showed that levels of phosphorylated Prkch were gradually decreased during OSKC (Oct4, Sox2, $\mathrm{Klf} 4$, and c-Myc)-induced reprogramming of control MEF cells, but not Rictor-knockdown MEF cells (Fig. 3d). Collectively, these data imply that Rictor facilitates phosphorylation of Prkch at Ser-642.

To further determine whether Rictor promotes ES cell differentiation via increasing Prkch phosphorylation, we first compared the abilities of wild-type Prkch, phosphorylation defective mutant Prkch S642A and phsphorylation mimics Prkch S642D to induce R1 ES cell differentiation. Similar to wild-type Prkch, Prkch S642D exhibited strong ability to induce R1 ES cell differentiation (Fig. 3e). However, Prkch S642A failed to show any effect on R1 ES cell differentiation (Fig. 3e). These data indicate that Prkch phosphorylation at Ser-642 is required for Prkch-induced ES cell differentiation. We next introduced Prkch S642A and Prkch S642D into Rictor-knockdown R1 ES cells. As was expected, knockdown of Rictor protected R1 ES cells from RA-induced differentiation (Fig. 3f). This effect of Rictor knockdown was reversed by ectopic expression of Prkch S642D, but not by Prkch S642A expression (Fig. 3f).
Taken together, these data suggest that Rictor promotes ES cell differentiation via facilitating Prkch phosphorylation.

\section{The Rictor-Prkch axis promotes ES cell differentiation via KIf4}

We next sought to examine how the Rictor-Prkch axis promotes ES cell differentiation. We found that knockdown of Klf4 led to the differentiation of Rictor-knockdown R1 ES cells under both RA-treated and -untreated conditions (Fig. 4a). In addition, expression of exogenous Klf4 inhibited R1 ES cell differentiation induced by Rictor (Fig. 4b). These data reveal Klf4 as a downstream mediator of Rictor in the regulation of ES cell differentiation. It has been previously shown that phosphorylation of Klf4 promotes ES cell differentiation [21]. We therefore asked whether Prkch could enhance Klf4 phosporylation and thus promote ES cell differentiation. To test this possibility, we first assessed levels of phosphorylated Klf4 in MEF cells expressing wild-type Prkch, phosphorylation mimics Prkch S642D or phosphorylation defective mutant Prkch S642A. Both wild-type Prkch and Prkch S642D were shown to increase levels of phosphorylated Klf4 (Fig. 4c). However, Prkch S642A failed to show any effect (Fig. 4c). These data demonstrate that Prkch is indeed able to enhance Klf4 phosphorylation. We next investigated whether Klf4 phosphorylation is prerequisite for Prkch-induced ES cell differentiation. By performing bioinformatics analysis, we found that five serine residues S110, S125, S133, S244 and S295 of Klf4 could be phosphorylated by Prkch. Interestingly, Klf4 S295A, but not wild-type Klf4, Klf4 S110A, Klf4 S125A, Klf4 S133A, and Klf4 S244A, was able to reverse Prkch-induced R1 ES cell differentiation (Fig. 4d). These combined data suggest that the Rictor-Prkch axis promotes ES cell differentiation through enhancing Klf4 phosphorylation at Ser-295.

\section{The Rictor-Prkch axis inhibits the transcriptional activity of KIf4 toward E-cadherin}

As a transcriptional factor, Klf4 has been shown to maintain ES cell identity by regulating various target genes expression. As a well-known Klf4 downstream target gene, Ecadherin is important for ES cell pluripotency maintenance, and downregulation of E-cadherin leads to ES cell differentiation. We therefore sought to examine whether the Rictor-Prkch axis promotes ES cell differentiation through inhibiting the transcriptional activity of Klf4 toward Ecadherin and decreasing E-cadherin expression. We first evaluated the effects of Rictor and Prkch on E-cadherin expression. Our data showed that ectopic expression of either Rictor or Prkch markedly decreased E-cadherin levels, whereas knockdown of their expression led to the 
Fig. 4 The Rictor-Prkch axis promotes ES cell differentiation through Klf4. a R1 cells expressing the indicated shRNAs were treated with 0.3 $\mu \mathrm{M}$ RA for 4 days. The ES cell colonies were then stained for alkaline phosphotase activity.

The shown images are representative from three replications. b R1 cells expressing the indicated proteins were treated with $0.3 \mu \mathrm{M}$ RA for 4 days. The ES cell colonies were then stained for alkaline phosphotase activity. The shown images are representative from three replications. c MEF cells were infected with lentiviruses expressing the indicated proteins. Forty-eight hours after infection, cell lysates were analyzed by Western blot. d $\mathrm{R} 1$ cells expressing the indicated proteins were treated with 0.3 $\mu \mathrm{M}$ RA for 4 days. The ES cell colonies were then stained for alkaline phosphotase activity.

The shown images are representative from three replications
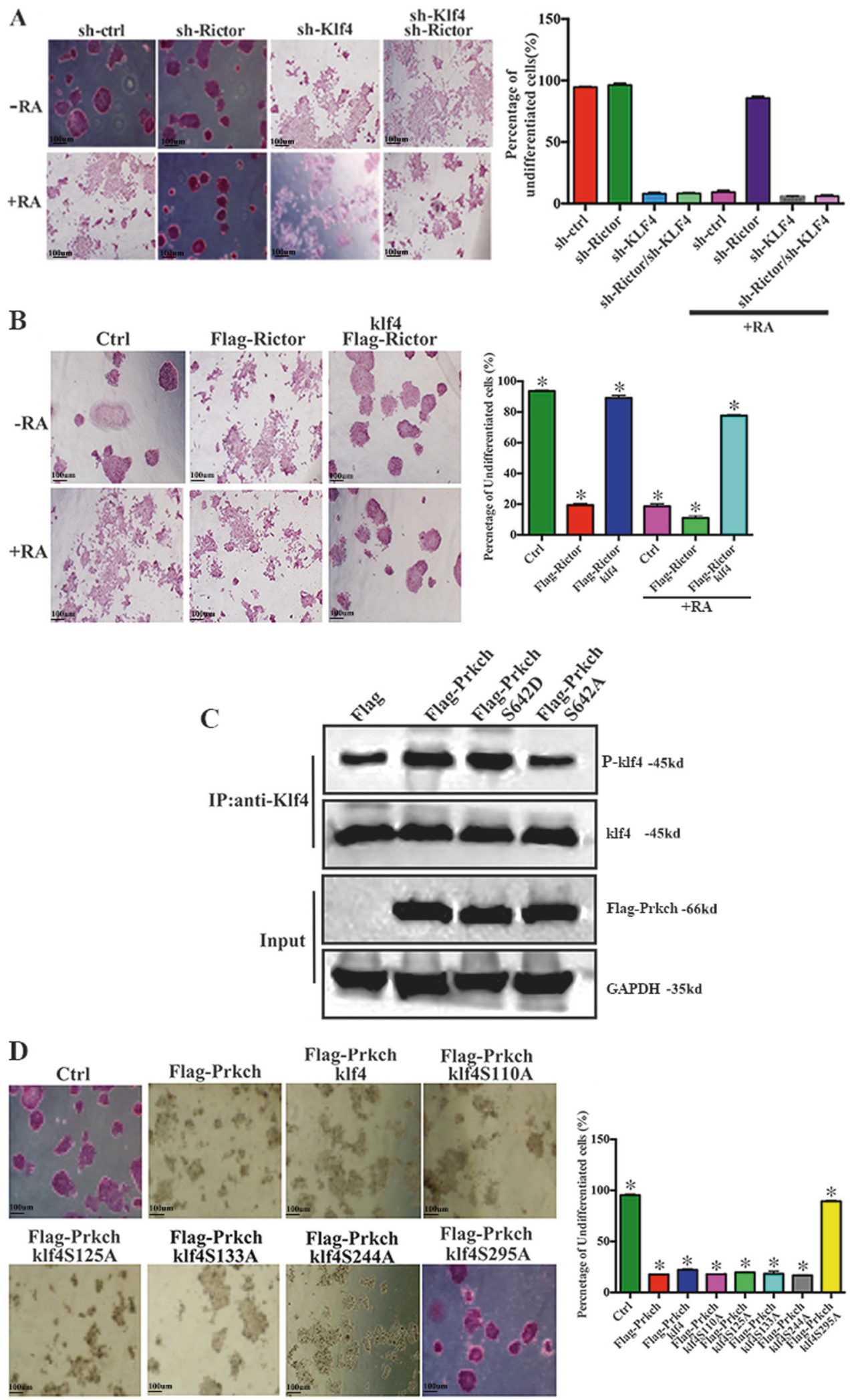

Flag-Prkch klf4S295A
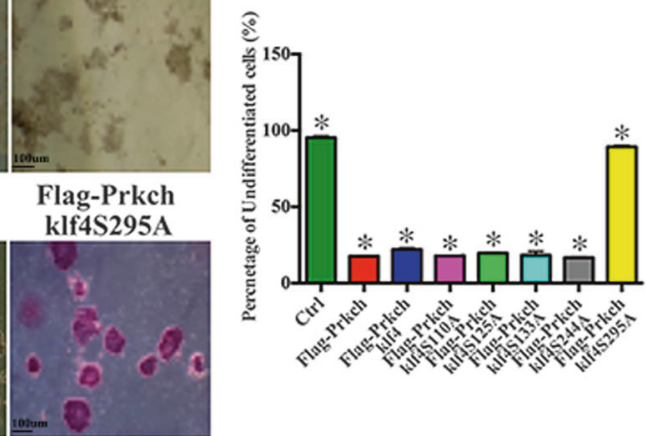

increased expression of E-cadherin (Fig. 5a, b). To further determine whether Rictor and Prkch regulate E-cadherin expression via Klf4, we examined the effect of Rictor on expression of E-cadherin in Prkch knockdown or Prkch and 
Fig. 5 The Rictor-Prkch axis decreases the transcriptional activity of Klf4 toward Ecadherin. a MEF cells were infected with lentiviruses expressing the indicated proteins or shRNAs. Forty-eight hours after infection, cell lysates were analyzed by western blot. b MEF cells were infected with lentiviruses expressing the indicated proteins or shRNAs. Forty-eight hours after infection, cell lysates were analyzed by western blot. c MEF cells were infected with lentiviruses expressing proteins or shRNAs as indicated. Forty-eight hours after infection, cell lysates were analyzed by western blot. d R1 cells expressing Flag-Prkch or control proteins were infected with control or Klf4 specific shRNAs as indicated. Fortyeight hours after infection, cell lysates were subjected to ChIP analysis with anti-Klf4 antibody or an isotype-matched IgG. ChIP products were amplified by semi-quantitative PCR. Actin was used as a negative control. e MEF cells were infected with lentiviruses expressing control, Prkch and Klf4 shRNAs in the indicated combination together with OSKC transduction. Fortyeight hours after infection, cell lysates were subjected to ChIP analysis with anti-Klf4 antibody or an isotype-matched IgG. ChIP products were amplified by semi-quantitative PCR. Actin was used as a negative control
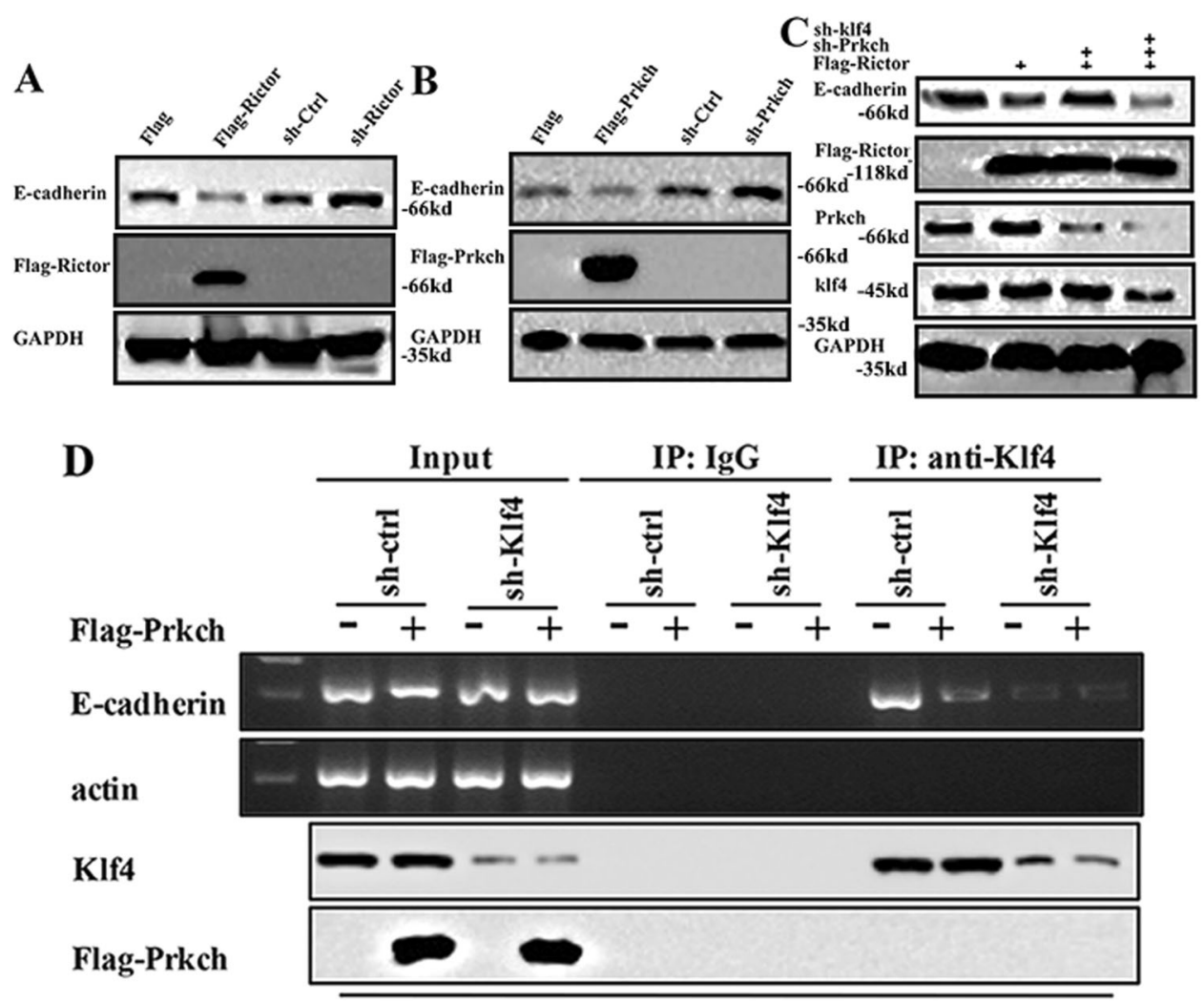

R1

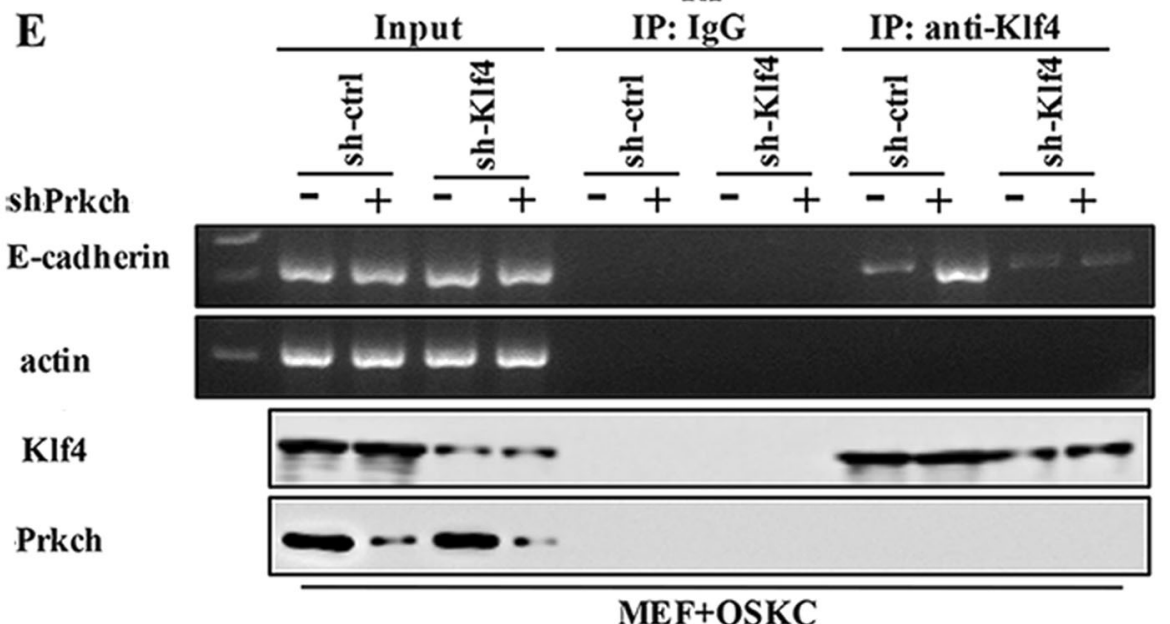

Klf4-double knockdown MEF cells. The results showed that Rictor-induced E-cadherin downregulation was greatly recovered by Prkch knockdown (Fig. 5c), reinforcing Prkch as a downstream effector of Rictor. Nonetheless, knockdown of Prkch failed to reverse Rictor-inhibited E-cadherin expression when Klf4 was simultaneously knocked down (Fig. 5c). These data indicate that the Rictor-Prkch axis regulates E-cadherin expression through Klf4.

We next wanted to determine how Prkch downregulates E-cadherin expression. The Chromatin immunoprecipitation (ChIP) assay showed that ectopic expression of Prkch was strongly decreased, whereas knockdown of Prkch greatly increased binding of Klf4 to E-cadherin promoter (Fig. 5d, e), indicating that Prkch inhibits Klf4-derived E-cadherin expression. The effect of Rictor has on the binding ability of Klf4 to the E-cadherin promoter was also been demonstrated (Figure S4A and B). Taken together, these data suggest that the Rictor-Prkch axis regulates ES cell differentiation via modulating Klf4 transcriptional activity. 


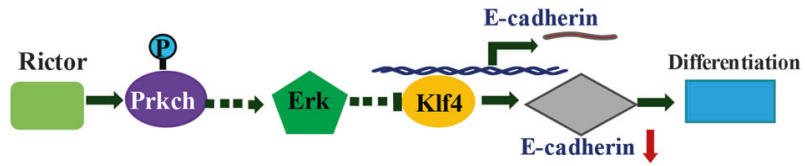

Fig. 6 The diagram of the pathway about Rictor-Prkch-Klf4 in ESCs

\section{Discussion}

mTOR is a central cell growth controller in response to nutrients and growth factors. mTOR nucleates two different multiprotein complexes, mTORC1 and mTORC2, which have distinct physiological functions. Although the cellular function of mTORC2 is less understood than that of mTORC1, it has been recently shown that mTORC2 plays an important role in the regulation of metabolic reprogramming, caner cell proliferation and invasion, and cancer drug resistance $[15,19,22]$. As a key component of mTORC2, Rictor is required for mTORC2 function. Therefore, it is not surprising to see dysregulation of Rictor is linked to a variety of human cancers [2325]. Here we demonstrate a novel and unexpected function of Rictor in association with mouse ES cell differentiation.

Rictor is shown to promote ES cell differentiation through Prkch. Via interaction with Prkch, Rictor promotes Prkch phosphorylation at Ser-642. More importantly, we show that Prkch phosphorylation is required for Rictor-induced ES cell differentiation. It has been previously reported that the Rictor-mTOR complex is able to phosphorylate both AKT and PKC $\alpha$ [18, 26, 27]. Since PI3K/Akt signaling pathway is involved in the maintenance of ES cell pluripotency [28-30], it would be interesting to determine whether Rictor could also regulate ES cell differentiation by modulating the PI3K/Akt pahway.

As a member of the PKC family proteins, Prkch has been reported to modulate ERK and JNK signaling pathways and regulate cell proliferation and differentiation [3134]. In this study, we show that Prkch is able to promote Klf4 phosphorylation and inhibit Klf4-dependent E-cadherin expression. Given that ERK-mediated Klf4 phosphorylation has been shown to suppress Klf4 activity and induce ES cell differentiation [21], we also found that overexpression of Prkch in the R1 ES cells could increase the phosphorylation of Klf4, and this phosphorylation of Klf4 decreased after the addition of ERK inhibitor PD98059 (Figure S5). it is conceivable that Prkch enhances Klf4 phosphorylation via ERK and thus promotes ES cell differentiation (Fig. 6). Taken together, our findings uncover a novel Rictor-Prkch-Klf4 pathway that is important for ES cell differentiation.

\section{Materials and methods}

\section{Cell culture}

Mouse embryonic stem cell line R1 was maintained in DMEM supplemented with $10 \%$ FBS, $10 \% \mathrm{KSR}, 2 \mathrm{mM}$ LGlutamine, $100 \mu \mathrm{M}$ Non Essential Amino acids (NEAA), $0.1 \mathrm{mM} \beta$-Mercaptoethanol, $1 \mathrm{mM}$ sodium pyruvate (Invitrogen) and $1000 \mathrm{U} / \mathrm{ml}$ LIF (Millipore). MEF and HEK 293T cells were maintained in DMEM supplemented with $10 \%$ FBS. The medium was replaced every $24 \mathrm{~h}$. The cells are collected directly after overnight culture due to the detection of p-Prkch and pKlf4.

\section{Protein overexpression in mouse ESCs}

To generate lentiviruses expressing the indicated proteins, HEK $293 \mathrm{~T}$ cells grown on a 6 -cm dish were transfected with $2 \mu \mathrm{g}$ of pSin-based construct, $1 \mu \mathrm{g}$ of pmd2.g, and $2 \mu \mathrm{g}$ of pspax2. Twelve hours after transfection, cells were cultured with DMEM medium containing 20\% FBS for an additional $24 \mathrm{~h}$. The culture medium containing lentivirus particles was filtered through a $0.45 \mu \mathrm{m}$ PVDF filter (Millipore) and incubated with mouse ESCs in the ESC culture medium supplemented with $4 \mu \mathrm{g} / \mathrm{Ml}$ polybrene (Sigma) for $12 \mathrm{~h}$, followed by selection with $5 \mu \mathrm{g} / \mathrm{ml}$ puromycin for another $24 \mathrm{~h}$.

\section{RNA interference}

To generate lentiviruses expressing shRNAs, HEK 293T cells grown on a 6-cm dish were transfected with $2 \mu \mathrm{g}$ of shRNAs (cloned in PLKO.1) or control vector, $2 \mu \mathrm{g}$ of pREV, $2 \mu \mathrm{g}$ of $\mathrm{pGag} / \mathrm{Pol} / \mathrm{PRE}$, and $1 \mu \mathrm{g}$ of pVSVG. Twelve hours after transfection, cells were cultured with DMEM medium containing $20 \%$ FBS for an additional $24 \mathrm{~h}$. The culture medium containing lentivirus particles was filtered through a $0.45 \mu \mathrm{m}$ PVDF filter (Millipore) and incubated with mouse ESCs supplemented with $4 \mu \mathrm{g} / \mathrm{ml}$ polybrene (Sigma) for $12 \mathrm{~h}$, followed by selection with $5 \mu \mathrm{g} / \mathrm{ml}$ puromycin for another $24 \mathrm{~h}$. The knockdown efficiency was evaluated by western blot analysis and real-time RT-PCR analysis. The shRNA sequences are as follows. shRNA rictor, CCGGCGAGACTTTGTCTGTCTAATTCTCGAGAATTAGACAGACAAAG TCTCGTTTTTG; shRNAPrkch, CCGGCC TAGAATCAAATCCCGAGAACT CGAGTTCTCGGGATTTGATTCTAGGTTTTT.

\section{Real-time RT-PCR}

For real-time RT-PCR analysis, total RNA was extracted using Trizol reagent (Sigma). cDNA synthesis was 
performed using a PrimeScript RT reagent kit following the manufacturer's instructions (Takara). Real-time PCR was performed using SYBR premix EX Taq (Takara) and ROX and analyzed with Stratagene Mx3000p (Agilent Technologies). Real-time PCR primer sequences are as follows: rictor $5^{\prime}$-ctgggctttaattatgaagac-3' and 5'-ctctccacctcgttgctctg$3^{\prime}$.

\section{Western blot analysis}

Western blot was performed as previously described [35]. The following antibodies were obtained from the indicated sources: the primary antibodies against GAPDH (Santa Cruz), Oct4 (Santa Cruz), Sox2 (Millipore), Rictor (Santa Cruz), Prkch (Abcam), p-Prkch (GenScript), E-cadherin (BD).

\section{Co-immunoprecipitation}

R1 cells were treated with $0.3 \mu \mathrm{M}$ retinoic acid (RA). Four days after treatment, cells were lysed with IP buffer $(20 \mathrm{mM}$ HEPES pH 7.4, 0.5 \% NP-40, 150 mM NaCl, 2 mM EDTA, $1.5 \mathrm{mM} \mathrm{MgCl2}$ ). The supernatant was incubated with either anti-Rictor antibody or an isotype-matched control $\mathrm{IgG}$ at 4 ${ }^{\circ} \mathrm{C}$ overnight. The immunoprecipitates were then subjected to western blot analysis with the indicated antibodies.

\section{Alkaline phosphatase staining}

Mouse ESCs were fixed in 4\% paraformaldehyde, rinsed two times with PBS and stained using the Alkaline Phosphatase Detection Kit (Millipore) according to the manufacturer's instruction.

\section{ChIP assay}

The ChIP assay was performed as previously described [36]. Briefly, cells were fixed with $1 \%$ formaldehyde and then sonicated to obtain soluble chromatin. After dilution, the chromatin solutions were incubated with protein A/GSepharose beads coated with anti-Klf4 antibody or an isotype-matched control IgG. After extensive washing of the beads, the bound DNA fragments was eluted and subjected to PCR amplification with the following primer pairs: 5'-TAGAGGGTCACCGCGTCTAT-3' and 5'-TCACAGGTGCTTTGCAGTT C-3'.

Acknowledgements This work was supported by the National Science Foundation of China (31501103, 81401518); the Anhui Province Funds for Distinguished Young Scientists (1508085J08); Key Projects of the Outstanding Young Talents in Colleges and Universities (gxyqZD2016058); the Anhui Provincial Natural Science Foundation (1508085QC62); Anhui Science and Technology Plan Project (1604a0802082, 1401045013), and the Doctoral Research Foundation of the Medical University of Anhui (0401062101).

Author contributions DHZ and YMZ conceived the idea; YMZ, GB, $\mathrm{CY}$ and $\mathrm{PW}$ wrote the manuscript; YMZ and LZ performed the experiments; YMZ and PW analyzed the data; JCH and YYW revised the manuscript; DHZ, GPZ and ZYZ designed the experiments and supervised the research.

\section{Compliance with ethical standards}

Conflict of interest The authors declare that they have no conflict of interest.

\section{References}

1. Yamanaka S, Blau HM. Nuclear reprogramming to a pluripotent state by three approaches. Nature. 2010;465:704-12.

2. Evans MJ, Kaufman MH. Establishment in culture of pluripotential cells from mouse embryos. Nature. 1981;292:154-6.

3. Thomson JA, Itskovitz-Eldor J, Shapiro SS, Waknitz MA, Swiergiel JJ, Marshall VS, et al. Embryonic stem cell lines derived from human blastocysts. Science. 1998;282:1145-7.

4. Matsuda T, Nakamura T, Nakao K, Arai T, Katsuki M, Heike T, et al. STAT3 activation is sufficient to maintain an undifferentiated state of mouse embryonic stem cells. EMBO J. 1999;18:4261-9.

5. Williams RL, Hilton DJ, Pease S, Willson TA, Stewart CL, Gearing DP, et al. Myeloid leukaemia inhibitory factor maintains the developmental potential of embryonic stem cells. Nature. 1988;336:684-7.

6. Ying QL, Nichols J, Chambers I, Smith A. BMP induction of Id proteins suppresses differentiation and sustains embryonic stem cell self-renewal in collaboration with STAT3. Cell. 2003;115:281-92.

7. Ying QL, Wray J, Nichols J, Batlle-Morera L, Doble B, Woodgett $\mathrm{J}$, et al. The ground state of embryonic stem cell self-renewal. Nature. 2008;453:519-23.

8. Chen X, Xu H, Yuan P, Fang F, Huss M, Vega VB, et al. Integration of external signaling pathways with the core transcriptional network in embryonic stem cells. Cell. 2008;133:1106-17.

9. Kim J, Chu J, Shen X, Wang J, Orkin SH. An extended transcriptional network for pluripotency of embryonic stem cells. Cell. 2008;132:1049-61.

10. Cornu M, Albert V, Hall MN. mTOR in aging, metabolism, and cancer. Curr Opin Genet Dev. 2013;23:53-62.

11. Laplante M, Sabatini DM. mTOR signaling in growth control and disease. Cell. 2012;149:274-93.

12. Kim DH, Sarbassov DD, Ali SM, King JE, Latek RR, ErdjumentBromage $\mathrm{H}$, et al. mTOR interacts with raptor to form a nutrientsensitive complex that signals to the cell growth machinery. Cell. 2002; 110:163-75.

13. Frias MA, Thoreen CC, Jaffe JD, Schroder W, Sculley T, Carr $\mathrm{SA}$, et al. mSin 1 is necessary for $\mathrm{Akt} / \mathrm{PKB}$ phosphorylation, and its isoforms define three distinct mTORC2s. Curr Biol. 2006;16:1865-70.

14. Tanaka K, Babic I, Nathanson D, Akhavan D, Guo D, Gini B, et al. Oncogenic EGFR signaling activates an mTORC2-NFkappaB pathway that promotes chemotherapy resistance. Cancer Discov. 2011;1:524-38.

15. Masui K, Cavenee WK, Mischel PS. mTORC2 in the center of cancer metabolic reprogramming. Trends Endocrinol Metab. 2014;25:364-73. 
16. Sarbassov DD, Ali SM, Kim DH, Guertin DA, Latek RR, Erdjument-Bromage $\mathrm{H}$, et al. Rictor, a novel binding partner of mTOR, defines a rapamycin-insensitive and raptor-independent pathway that regulates the cytoskeleton. Curr Biol. 2004; 14:1296-302.

17. Pearce LR, Huang X, Boudeau J, Pawlowski R, Wullschleger S, Deak M, et al. Identification of Protor as a novel Rictor-binding component of mTOR complex-2. Biochem J. 2007;405: 513-22.

18. Sarbassov DD, Guertin DA, Ali SM, Sabatini DM. Phosphorylation and regulation of Akt/PKB by the rictor-mTOR complex. Science. 2005;307:1098-101.

19. Zhang F, Zhang X, Li M, Chen P, Zhang B, Guo H, et al. mTOR complex component Rictor interacts with PKCzeta and regulates cancer cell metastasis. Cancer Res. 2010;70:9360-70.

20. Shiota C, Woo JT, Lindner J, Shelton KD, Magnuson MA. Multiallelic disruption of the rictor gene in mice reveals that mTOR complex 2 is essential for fetal growth and viability. Dev Cell. 2006;11:583-9.

21. Kim MO, Kim SH, Cho YY, Nadas J, Jeong CH, Yao K, et al. ERK1 and ERK2 regulate embryonic stem cell self-renewal through phosphorylation of Klf4. Nat Struct Mol Biol. 2012;19:283-90.

22. Masui K, Cavenee WK, Mischel PS. mTORC2 dictates Warburg effect and drug resistance. Cell Cycle. 2014;13:1053-4.

23. Tang F, Wu Q, Ikenoue T, Guan KL, Liu Y, Zheng P. A critical role for Rictor in T lymphopoiesis. J Immunol. 2012;189:1850-7.

24. Wazir U, Newbold RF, Jiang WG, Sharma AK, Mokbel K. Prognostic and therapeutic implications of mTORC1 and Rictor expression in human breast cancer. Oncol Rep. 2013;29:1969-74.

25. Wen SY, Li CH, Zhang YL, Bian YH, Ma L, Ge QL, et al. Rictor is an independent prognostic factor for endometrial carcinoma. Int J Clin Exp Pathol. 2014;7:2068-78.

26. Ikenoue T, Inoki K, Yang Q, Zhou X, Guan KL. Essential function of TORC2 in PKC and Akt turn motif phosphorylation, maturation and signalling. EMBO J. 2008;27:1919-31.
27. Guertin DA, Stevens DM, Thoreen CC, Burds AA, Kalaany NY, Moffat $\mathbf{J}$, et al. Ablation in mice of the mTORC components raptor, rictor, or mLST8 reveals that $\mathrm{mTORC} 2$ is required for signaling to Akt-FOXO and PKCalpha, but not S6K1. Dev Cell. 2006;11:859-71.

28. Niwa H, Ogawa K, Shimosato D, Adachi K. A parallel circuit of LIF signalling pathways maintains pluripotency of mouse ES cells. Nature. 2009;460:118-22.

29. Paling NR, Wheadon H, Bone HK, Welham MJ. Regulation of embryonic stem cell self-renewal by phosphoinositide 3-kinasedependent signaling. J Biol Chem. 2004;279:48063-70.

30. Takahashi K, Murakami M, Yamanaka S. Role of the phosphoinositide 3-kinase pathway in mouse embryonic stem (ES) cells. Biochem Soc Trans. 2005;33:1522-5.

31. Uht RM, Amos S, Martin PM, Riggan AE, Hussaini IM. The protein kinase $\mathrm{C}$-eta isoform induces proliferation in glioblastoma cell lines through an ERK/Elk-1 pathway. Oncogene. 2007;26:2885-93.

32. Kashiwagi M, Ohba M, Chida K, Kuroki T. Protein kinase C eta (PKC eta): its involvement in keratinocyte differentiation. J Biochem. 2002;132:853-7.

33. Brandlin I, Hubner S, Eiseler T, Martinez-Moya M, Horschinek A, Hausser A, et al. Protein kinase C (PKC)eta-mediated PKC mu activation modulates ERK and JNK signal pathways. J Biol Chem. 2002;277:6490-6.

34. Rotem-Dai N, Oberkovitz G, Abu-Ghanem S, Livneh E. PKCeta confers protection against apoptosis by inhibiting the proapoptotic JNK activity in MCF-7 cells. Exp Cell Res. 2009;315:2616-23.

35. Zhu Y, Yao Z, Wu Z, Mei Y, Wu M. Role of tumor necrosis factor alpha-induced protein 1 in paclitaxel resistance. Oncogene. 2014;33:3246-55.

36. Jin L, Hu WL, Jiang CC, Wang JX, Han CC, Chu P, et al. MicroRNA-149*, a p53-responsive microRNA, functions as an oncogenic regulator in human melanoma. Proc Natl Acad Sci USA. 2011;108:15840-5. 